\title{
IdeAs
}

Idées d'Amériques

$17 \mid 2021$

Villes et culture dans les Amériques

\section{La lutte juridique contre les assassinats des leaders sociaux en Colombie}

Rodrigo Uprimny-Yepes et Laetitia Braconnier-Moreno

\section{OpenEdition}

1 Journals

Édition électronique

URL : https://journals.openedition.org/ideas/10030

DOI : $10.4000 /$ ideas. 10030

ISSN : $1950-5701$

Éditeur

Institut des Amériques

Référence électronique

Rodrigo Uprimny-Yepes et Laetitia Braconnier-Moreno, «La lutte juridique contre les assassinats des leaders sociaux en Colombie », IdeAs [En ligne], 17 | 2021, mis en ligne le 01 mars 2021, consulté le 04 juin 2021. URL : http://journals.openedition.org/ideas/10030 ; DOI : https://doi.org/10.4000/ideas. 10030

Ce document a été généré automatiquement le 4 juin 2021.

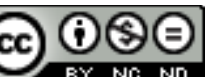

IdeAs - Idées d'Amériques est mis à disposition selon les termes de la licence Creative Commons Attribution - Pas d'Utilisation Commerciale - Pas de Modification 4.0 International. 


\title{
La lutte juridique contre les assassinats des leaders sociaux en Colombie
}

\author{
Rodrigo Uprimny-Yepes et Laetitia Braconnier-Moreno
}

\section{INTRODUCTION}

1 L' «action de tutelle» (tutela), présentée conjointement le 10 décembre 2019 par plusieurs mouvements sociaux et organisations de défenses des droits humains, a pour objet de demander la déclaration d'un «État de choses inconstitutionnel », concernant la violence exercée à l'encontre des leaders sociaux. La notion d' «État de choses inconstitutionnel», créée par la jurisprudence colombienne, fait référence aux situations dans lesquelles, sur la base d'une action judiciaire individuelle telle que la tutela - protection spéciale des droits fondamentaux -, la Cour constate que la violation d'un droit n'est pas d'ordre individuel, mais s'inscrit dans un contexte de violation massive des droits humains, qui exige des réponses telles que des politiques publiques structurelles (Uprimny-Yepes, R., 2007).

2 Au nom de dix leaders sociaux - activistes et défenseurs environnementaux, paysans, autochtones, étudiants, victimes du conflit armé - la tutelle a ainsi sollicité :

3 - que soient prises non seulement des mesures de protection physique, mais aussi de prévention qui garantissent le droit de défendre les droits humains, y compris des mesures politiques contre la stigmatisation des leaders sociaux ;

4 - des mécanismes de protection basés sur une évaluation des risques provenant des territoires et construites avec la participation directe du leader en danger, afin que ces mesures soient efficaces ;

5 - des garanties de sécurité qui matérialisent les approches différentielles, ethnoraciales et de genre ; et 
- des outils de protection collective et pas seulement individuelle, articulés avec les initiatives d'autoprotection. Pour cela, il est demandé que le Gouvernement mette en œuvre les dispositifs prévus pour cette protection collective des leaders sociaux dans l'Accord de Paix.

7 Ce phénomène de violence résulte d'un constat empirique ancien et revêt, $a$ fortiori depuis la signature de l'Accord de Paix, un caractère systématique (1). Ce caractère systématique a bel et bien des conséquences dans la construction de mécanismes de protection adéquats. Encore faut-il analyser les facteurs explicatifs de ces assassinats ciblés, à savoir l'insuffisance de la présence institutionnelle dans les territoires anciennement contrôlés par la guérilla, la précarité de la démocratie colombienne, les conflits socio-économiques liés, entre autres, à l'usage de la terre (2). Ce n'est qu'au moyen de cette reconnaissance que la réponse gouvernementale sera à la hauteur de la gravité de la situation (3).

\section{Élément empirique : la persistance et l'aggravation de la violence}

8 Pour illustrer la persistance de ce type de violence, soulignons que dès 1998, dans son arrêt T-590, la Cour constitutionnelle colombienne a déclaré un «état de chose inconstitutionnel» en raison du manque de protection de la vie des défenseurs des droits humains. Pour sa part, le bureau du Haut-Commissariat des Nations Unies aux droits de l'homme de Bogota a pointé le manque de garanties pour la défense des droits de l'homme dans tous ses rapports annuels depuis 1997, et signale qu'il s'agit de l'un des plus graves problèmes auxquels est confrontée la Colombie.

9 Pourtant, la situation a encore empiré depuis la signature de l'Accord de paix. L'organisation Somos Defensores, par exemple, documente l'ampleur de la violence contre les activistes et les défenseurs depuis quinze ans. Son rapport publié en 2020 montre une évolution alarmante entre l'année 2010, qui enregistrait 174 cas d'agressions contre des leaders sociaux, dont 32 meurtres, et l'année 2019, au cours de laquelle 844 cas de violence, dont 124 meurtres, ont pu être recensés.

10 Ces agressions sont commises par différents types d'acteurs: des acteurs illégaux héritiers du paramilitarisme, tels que les « Aguilas negras » et les Autodefensas gaitanistas de Colombia, les dissidents des ex-FARC, et parfois de l'ELN (Ejercito de liberación nacional) et de l'EPL (Ejercito popular de liberación); mais aussi des acteurs légaux, membres de l'armée. De nombreuses affaires ne sont pas résolues et les auteurs intellectuels de ces crimes bénéficient d'un niveau d'impunité élevé.

11 En tout état de cause, cette violence est de nature systématique au regard des leaders visés. Il existe ainsi des similitudes entre leurs revendications : nombre d'entre eux cherchent l'application territoriale de l'Accord de paix, et en particulier la substitution des cultures illicites par d'autres opportunités pour les paysans dans le cadre du Programme national intégral de substitution des cultures illicites (PNIS) et la formulation des Plans de développement avec approche territoriale (PDET); d'autres sont impliqués dans les procédures de restitution des terres spoliées au cours du conflit; d'autres, enfin, dénoncent la présence d'acteurs armés et le recrutement d'enfants et d'adolescents dans les périphéries des villes et les zones rurales - 
principales zones affectées, avec une forte concentration dans les départements du Cauca et d'Antioquia.

La plupart de ces leaders entretiennent un lien étroit avec leur territoire, en particulier ceux qui agissent pour la garantie des droits territoriaux des peuples autochtones et afro-colombiens, et pour la préservation des ressources naturelles. Alors que ces éléments sont connus, comment expliquer la continuité de ces assassinats systématiques? Il s'agit d'un débat complexe, mais certaines faiblesses de l'État colombien peuvent être mises en évidence.

\section{Les facteurs explicatifs de cette violence ciblant les leaders sociaux}

13 L'une de ces faiblesses concerne la quasi-absence de l'État sur de nombreux territoires. Or, il était prévisible qu'en déposant les armes, la guérilla des FARC laisserait un vide de pouvoir susceptible d'attirer d'autres groupes armés, et que, par conséquent, l'État devrait "récupérer ces territoires" au moyen de l'ensemble de son arsenal institutionnel. Pour autant, cette récupération n'a pas eu lieu, qui explique la dispute entre les acteurs armés susmentionnés pour le contrôle territorial.

Les régions aujourd'hui affectées par la violence sont ainsi caractérisées par un faible niveau de développement, entrainant la persistance d'économies illégales. Dans ce contexte, les leaders locaux, souvent déjà victimes du conflit armé, se retrouvent contraints à résister aux exactions commises par les groupes armés: recrutements forcés et exploitation sexuelle de mineur.e.s, déplacements forcés, confinements de population sur les routes du narcotrafic, extorsions, etc.

15 Il est essentiel de souligner de surcroît la précarité de la démocratie colombienne. L'histoire récente montre que chaque ouverture démocratique au niveau national a été suivie d'une sorte de fermeture violente dans les territoires. Au début des années 1980, par exemple, sous la présidence de Betancur, les efforts d'ouverture du pays ont été accompagnés de l'émergence d'un paramilitarisme régional, parfois de manière complice avec les forces de l'ordre.

16 L'histoire semble se répéter: des "circonscriptions électorales spéciales", devant favoriser la participation dans l'arène politique de populations qui en ont été historiquement exclues, ont été prévues par l'Accord de paix. Ainsi, les dernières élections régionales en Colombie ont enregistré un fort taux d'inscription de candidats d'origine paysanne, afro-colombienne et autochtones. Cependant, sur $36 \%$ des municipalités du pays a pesé la menace de ne pas pouvoir tenir des élections libres, du fait du contrôle exercé par des groupes armés sur les territoires (Defensoría del pueblo, 2019). Entre le $1^{\mathrm{er}}$ janvier et le 15 août 2019, 147 candidats, pré-candidats et membres d'organisations politiques, et 52 leaders sociaux ont fait l'objet de conduites portant atteinte à leurs droits.

17 Le fait, pour les leaders issus de ces populations, de se prononcer en faveur de la réforme rurale prévue par l'Accord ou du programme de substitution des cultures illicites, est ainsi générateur de risque. Cette situation est symptomatique de l'existence d'un intérêt politique et social pour le maintien du statu quo, attaché aux intérêts économiques de grands investisseurs. 
18 En effet, des problématiques socio-économiques non résolues persistent, comme celles liées à la concentration des terres. Au sein des territoires riches en ressources naturelles, des populations sont fréquemment contraintes de se déplacer, non plus du fait du conflit armé, mais plutôt à cause des activités des entreprises et de l'État en lien avec l'exploitation de la nature. En ce sens, selon le Rapporteur sur la situation des défenseurs des droits humains, beaucoup de communautés colombiennes sont négativement affectées par les projets des entreprises internationales et nationales, publiques et privées (Rapporteur spécial sur la situation des défenseurs et défenseuses des droits de la personne, 2020). Au-delà de l'inaction de l'État, de son apparente connivence avec les groupes armés illégaux, et de la corruption de corps de sécurité de l'État, sont dénoncés des « achats de sécurité » réalisés par des entreprises ${ }^{1}$.

Enfin, la forte stigmatisation des leaders sociaux - considérés comme des opposants gênant les méga projets de développement ou d'extraction des sols, ou pire, assimilés aux guérilleros et trafiquants de drogue - a pu être tolérée par un gouvernement indolent. Cette stigmatisation est lourde de conséquences, dans la mesure où elle contribue à légitimer les exactions commises à l'encontre des leaders.

\section{La réponse inadéquate du gouvernement actuel}

20 Face à l'augmentation de ces agressions, le diagnostic conduit par le gouvernement est erroné. Celui-ci revient à attribuer les responsabilités aux seules économies illicites et aux acteurs armés en marge de la loi, sans reconnaître la responsabilité de l'État. Il insiste, de plus, à instaurer un nouveau programme pour affronter cette criminalité, en laissant de côté les mécanismes créés aux termes des négociations entre le gouvernement et la guérilla des FARC sur la manière de traiter cette violence exacerbée contre les leaders sociaux ${ }^{2}$. Ces instruments devaient notamment permettre de passer de la protection individuelle des leaders, nécessaire mais insuffisante, à un système de garantie collective.

21 Abandonnant ces outils négociés, le gouvernement a lancé le PAO (Plan de Acción Oportuna). Il a ainsi ignoré le fait que la multiplication de stratégies parallèles, méconnaissant l'Accord de paix, rend plus difficile la mise en place de garanties effectives pour la sécurité des leaders. Dans le même temps, il a invoqué la diminution des attaques des leaders sociaux en falsifiant les chiffres du Haut-Commissaire des Nations-Unies, tout comme l'a d'ailleurs fait le conseiller présidentiel pour les droits humains de l'époque, et maintenant Fiscal General ${ }^{3}$, Francisco Barbosa - un diplômé des hautes universités françaises, que sa formation en France n'a pas aidé à s'abstenir de fausser les statistiques sur la violence contre les leaders sociaux; la réduction significative des assassinats annoncée n'est en effet pas conforme à la réalité.

$\mathrm{Au}$ vu des faiblesses de la réponse gouvernementale, la présentation d'une tutela s'est avérée nécessaire, comme recours devant les instances judiciaires nationales pour protéger les droits des défenseurs. Cette dernière a déjà fait l'objet d'un jugement favorable de la part du juge de première instance et du tribunal de Bogota dans sa décision du 25 mars 2020.

Cette décision est fondamentale en ce qu'elle reconnait, en premier lieu, le manquement systématique de l'État à son devoir de protéger les leaders sociaux et demande une protection intégrale afin qu'ils puissent exercer le droit de défendre les 
droits, essence même de leur activité. En second lieu, elle ordonne l'adoption de politiques publiques qui permettent de matérialiser les droits des leaders - au-delà d'une déclaration de principes. Ces dernières impliquent l'activation des protocoles d'analyse des risques et réactivent les espaces de concertation sur ces politiques. La décision reconnait l'importance des dimensions individuelle et collective des facteurs de risque au niveau régional et national. Elle prévoit, enfin, des mécanismes judiciaires de suivi du respect et de la mise en œuvre de cette décision.

Cette Cour enjoint donc à l'État colombien, et tout particulièrement au gouvernement, d'adopter des mesures de garanties spécifiques, non seulement pour la protection individuelle des leaders menacés, mais aussi pour assurer collectivement le labeur de tous les leaders sociaux. En outre, le gouvernement est sommé d'agir rapidement, et ce indépendamment de l'urgence sanitaire liée à la COVID-19. D'une énorme importance est certainement la mesure appelant les autorités à promouvoir un changement d'opinion dans la société, de manière à ce que la majorité comprenne et reconnaisse le travail crucial de ces leaders.

\section{Conclusions}

L'enjeu est ainsi posé : il consiste en ce que le gouvernement accepte l'erreur de son diagnostic et mette en place les systèmes de protection prévus dans le cadre de l'Accord de paix. L'espoir réside aussi dans le fait que la décision du tribunal de Bogota soit, dans le futur, entérinée par la Cour constitutionnelle colombienne.

En parallèle, la communauté internationale peut exercer une pression importante sur le bureau du Fiscal Général. Il semblerait nécessaire que ce dernier prenne au sérieux les analyses académiques justifiant des failles dans les politiques étatiques actuelles. Ces études montrent que la manière d'enquêter sur ces crimes doit être modifiée, en les hiérarchisant de façon appropriée et en reconnaissant son caractère systématique, afin d'obtenir des résultats, tout en identifiant les acteurs intellectuels de ces crimes.

Finalement, si la tutela et cet article portent principalement sur la violence dirigée contre les leaders sociaux, notre analyse et nos conclusions s'appliquent, mutatis mutandis, à la terrible violence exercée contre les ex-guérilleros des ex-FARC qui se sont démobilisés. Cette violence est d'une ampleur tout aussi inacceptable: plus de 240 d'entre eux ont été assassinés entre la signature de l'Accord de paix (novembre 2016) et la fin de l'année 2020. Le gouvernement Duque maintient aussi envers ce phénomène une attitude très problématique : il l'attribue uniquement à des groupes armés privés, tout en niant toute responsabilité de l'État s'agissant de ce phénomène. Plus grave encore, il se résiste aussi à appliquer les instruments prévus dans l'Accord de Paix pour y faire face. Nos recommandations concernant la violence envers les leaders sociaux sont donc aussi applicables aux assassinats des ex-guérilleros démobilisés. 


\section{BIBLIOGRAPHIE}

Defensoría del pueblo, « Alerte précoce 035-19 sur le risque électoral » pour la période électorale du 27 octobre 2019.

Gouvernement national de Colombie, FARC-EP et pays garants, Accord Final pour la fin du conflit et la construction d'une paix stable et durable, 24 novembre 2016.

Michalowski Sabine, Sanchez León, Nelson Camilo, Marin Daniel et al. Entre coacción y colaboración. Verdad judicial, actores económicos y conflicto armado en Colombia, 2018, Dejusticia

Rapport du Rapporteur spécial sur la situation des défenseurs et défenseuses des droits de la personne (A/HRC/43/51/Add.1) : 43ํㅜㄹiode de Session du Conseil des Droits Humains (FévrierMars 2020), disponible à l'adresse https://news.un.org/es/story/2020/03/1470571, consulté le 9 février 2021.

Uprimny-Yepes, Rodrigo, «10. La justice au cœur du politique : potentialités et risques d'une judiciarisation en Colombie ", dans Jacques Commaille éd., La fonction politique de la justice. Paris, La Découverte, « Recherches/Territoires du politique », 2007, p. 229-250.

\section{NOTES}

1. Plusieurs rapports d'analyses et d'organismes de défense des droits humains documentent ces collaborations. Voir par exemple: Michalowski, S., Sanchez León, N. C, Marin, D. et alt. Entre coacción y colaboración. Verdad judicial, actores económicos y conflicto armado en Colombia, 2018 , Dejusticia

2. Point 3.4 de l'Accord de paix. Garanties de sécurité: série d'instruments et de mesures pour la protection des communautés, leaders et défenseurs des droits humains et le démantèlement des organisations paramilitaires et criminelles.

3. Le «Fiscal General » en Colombie n'a pas d'équivalent institutionnel en France. C'est le responsable de l'institution qui coordonne les enquêtes criminelles et qui doit accuser les possibles responsables auprès des juges pénaux. Il ressemble à l'Attorney General des États-Unis, à la différence qu'il est indépendant du gouvernement.

\section{AUTEURS}

\section{RODRIGO UPRIMNY-YEPES}

Rodrigo Uprimny-Yepes est membre fondateur, ancien Directeur et chercheur de l'organisation Dejusticia. Il est avocat, titulaire d'une maitrise (DEA) en sociologie du développement de l'Université de Paris I (IEDES) et d'un doctorat en économie de l'Université d'Amiens. Professeur émérite à l'Université nationale de Colombie et professeur invité dans plusieurs universités. Il a été juge auxiliaire de la Cour constitutionnelle colombienne et est "co-juge" de cette Cour et du Conseil d'État. Il est membre de la Commission Internationale des juristes et du Comité des 
Nations-unies sur les droits économiques, sociaux et culturels (Comité DESC) pour la période 2015-2022.

\section{LAETITIA BRACONNIER-MORENO}

Laetitia Braconnier Moreno est représentante du pôle Bogota de l'Institut des Amériques. Avocate de l'Université Pontificale Bolivarienne (Medellin) et diplômée d'un Master 2 Droits humains de l'Université Paris Nanterre, elle est Doctorante en cotutelle entre cette Université (UMR 70/74, CREDOF) et l'Université Nationale de Colombie (EILUSOS). Elle est coprésidente de la commission « justice transitionnelle » de l'Association des juristes francocolombiens, et ses travaux portent notamment sur les droits des personnes migrantes et des groupes ethniques, et le pluralisme juridique. lbraconnier.moreno@gmail.com. 